\title{
CAP2 contributes to tumorigenesis in gastric cancer by targeting transcription factor SOX9
}

\author{
Ying Wan ${ }^{1 \#}$, Shengkui Qiu ${ }^{2 \#}$, Lei Yin ${ }^{2 \#}$, Xuesong Gao ${ }^{2}$, Yasu Jiang ${ }^{2}$, Shichun Feng ${ }^{2}$, Chong Tang ${ }^{2}$ \\ ${ }^{1}$ Department of Geratology, Affiliated Hospital 2 of Nantong University, Nantong, China; ${ }^{2}$ Department of General Surgery, Affiliated Hospital 2 of \\ Nantong University, Nantong, China \\ Contributions: (I) Conception and design: C Tang, S Feng; (II) Administrative support: Y Wan; (III) Provision of study materials or patients: S Qiu, \\ L Yin; (IV) Collection and assembly of data: X Gao; (V) Data analysis and interpretation: Y Jiang; (VI) Manuscript writing: All authors; (VII) Final \\ approval of manuscript: All authors. \\ \#These authors contributed equally to this work. \\ Correspondence to: Chong Tang; Shichun Feng. Department of General Surgery, Affiliated Hospital 2 of Nantong University, 6 North Haierxiang \\ Road, Nantong 226000, China. Email: 15051273108@163.com; fengshichungs@sina.com.
}

Background: Gastric cancer (GC) is one of the most common tumors and the major cause of cancerrelated mortality in the world. The purpose of this study is to identify new biomarker and reveal its potential molecular mechanism in GC.

Methods: The expression of CAP2 was observed by the bioinformatics analysis and western blot assays. The effects of CAP2 on cell proliferation and growth were tested by MTT assay, EdU assay, colony formation assay, and flow cytometric assay, respectively. ChIP and dual-luciferase assays were confirmed that SOX9 binding sites were putative regulatory elements in the transcriptional activation of CAP2. Furthermore, western blot and xenograft assays were applied to examine whether SOX9 was involved in the regulation of CAP2 expression.

Results: We reported that CAP2 is overexpressed in GC cells and tissues and related to a poorer prognosis for GC patients. Moreover, we found that knockdown of CAP2 suppressed the proliferation, growth, and cell cycle of GC cells. Besides, the transcription factor SOX9 participated in the CAP2-mediated proliferation of GC cells in vitro and in vivo.

Conclusions: Our results provide novel evidence that CAP2 plays an essential role in the genesis and development of GC, thus potentially highlighting this gene as a therapeutic target.

Keywords: CAP2; gastric cancer (GC); cell proliferation; SOX9

Submitted Jun 12, 2020. Accepted for publication Nov 17, 2020.

doi: 10.21037/jgo-20-234

View this article at: http://dx.doi.org/10.21037/jgo-20-234

\section{Introduction}

Gastric cancer (GC) is part of the main causes of cancerrelated death in the world (1). Over the last decades, despite advances in the surgical techniques and therapeutic strategies, the 5-year survival rate of severe GC patients is still very low (2), and one of the reasons for the high mortality rate is that most patients are diagnosed with advanced tumor grades and cancer metastasis, losing the optimal treatment time (3). Moreover, genetic mutations, epigenetic changes, and abnormal molecular signaling pathways are implicated in the occurrence, spread, and metastasis of GC (4). Thus, understanding the pathogenesis of GC at the molecular level and identifying potential molecular targets in the process of metastasis is important for diagnosis, prognosis, and GC therapy.

Cyclase-associated proteins (CAPs) are evolutionally conserved proteins that belong to the actin monomerbinding protein family and were thought to regulate actin cytoskeleton assembly (5). Accumulating evidence 
reveals that the actin cytoskeleton can play vital roles in cell morphogenesis, cytokinesis, and cell migration, and an abnormal actin cytoskeleton is usually the basis of tumorigenesis and metastasis (6). Therefore, CAPs play an important role in cell division and cell motility through its action on actin filament dynamics and the cytoskeleton. Previously, we reported that CAP2, a homolog of CAP (7), has been implicated in several types of cancers, including hepatocellular carcinoma (8), malignant melanoma (9), epithelial ovarian cancer (10), breast cancer (11), glioma (12), however, the biological function and precise mechanism of CAP2 in GC are not understood.

Herein, we aimed to estimate the role of CAP2 in GC, and then we further evaluated the molecular mechanisms whereby CAP2 could influence GC through in vitro and in vivo experiments in which we estimated the functions of CAP2 on cell proliferation. In addition, we showed that CAP2 is transcriptionally activated by SOX9, while SOX9 was proved to mediate the CAP2-regulated cell proliferation of GC in vitro and tumor growth in vivo. Finally, the above results supported an oncogenic activity for CAP2 in GC tumorigenesis, which can act as a therapeutic potential biomarker in GC.

We present the study in accordance with the ARRIVE reporting checklist (available at http://dx.doi.org/10.21037/ jgo-20-234).

\section{Methods}

\section{Clinical specimens and cell culture}

Fresh GC tissues and corresponding peritumor tissues were obtained from 24 patients who experienced surgical resection at the Affiliated Hospital of Nantong University. None of these patients received any preoperative anticancer treatment. This study was conducted in accordance with the Declaration of Helsinki (as revised in 2013), and was approved by Ethics the Committee of Affiliated Hospital 2 of Nantong University (ID: 2020KT042). Informed consent was provided by all participants.

The MKN45, BGC823, AGS, and SGC7901 GC cell lines and gastric epithelial cell (GES) line were obtained from the Chinese Academy of Sciences. These cell lines were cultured with DMEM containing $10 \%$ FBS in a humidified incubator at 377 with $5 \% \mathrm{CO}_{2}$.

\section{Transfection}

Specific or scrambled siRNAs (GenePharma) were applied to transfect cell lines by Lipofectamine 2000 (Invitrogen). The targeting sequence of CAP2 and SOX9 was as follows: CAP2-siRNA1: CCTGTAGCATCCACAGTAT, CAP2-siRNA2: GCGCCAAGTCATCTGAAAT, CAP2siRNA3: GGATGGTGATTATAGAGAA, siSOX9: GCAGCGACGUCAUCUCCAA.

\section{Western blot analysis}

Tissues and cells were lysed using RIPA buffer (Promega). Proteins were separated using SDS-PAGE and transferred into a PVDF membrane. Subsequently, the membranes were blocked for $2 \mathrm{~h}$ with $5 \%$ non-fat milk and then incubated with primary antibodies CAP2 (ab236590, Abcam)and SOX9 (ab3697, Abcam) at $4{ }^{\circ} \mathrm{C}$ overnight. Then, horseradish peroxidase-conjugated secondary antibodies were used to incubate the membrane at room temperature for 2 hours after three washes with TBST. The protein bands were visualized by ECL kits (Millipore, MA, USA).

\section{MTT assay}

Cells were grown in 96-well plates $\left(2 \times 10^{3}\right.$ cells/well) for $24 \mathrm{~h}$. Cell viability and proliferation assay were carried out using the MTT kit (Beyotime) after transfection or CellLight ${ }^{\mathrm{TM}}$ EdU Apollo567 in vitro kit (Ribo). For the MTT assay, the absorbance was measured at $490 \mathrm{~nm}$. For the EdU assay, positive cells in each group were recorded under the fluorescence microscope.

\section{Colony formation assay}

Cells were plated in a 12 -well plate at a density of $1 \times 10^{3}$ cells/well, with media changed every third day. After two weeks, cells were fixed with methanol and stained with crystal violet (Sigma). Subsequently, the number of colonies was counted.

\section{Flow cytometric analysis}

The cells were collected and fixed. Cell apoptosis was stained through using Annexin V- FITC/propidium iodide 
(PI) apoptosis kit (Invitrogen) and the percentage of cells was stained via a PI-based cell cycle kit (Invitrogen). The stained cells were detected by Attune Acoustic Focusing Cytometer (Invitrogen).

\section{Chromatin immunoprecipitation assay (ChIP)}

In brief, the protein-DNA complexes were cross-linked with $1 \%$ formaldehyde, followed by nuclear fractionation and DNA shearing by sonication. The immunoprecipitation was applied with an anti-SOX9 antibody (ab3697, Abcam) or IgG (negative control). Then, the antibodyprotein-DNA complex was eluted from the beads and reversed cross-linked after washing. PCR primers: Site1-F: 5'-TGTGGCTCATCCACTCTTAG-3', Site1-R: 5'-TTCCAAGCATCCATTCATAT-3', Site2-F: 5 '-GATAGGGAGGGTGCTGG-3', Site2-R: 5'-GCTGACAGTAATGAGGTGCA-3', Site3-F: 5'-TATTGGCCTTTAAGAAATTC-3', Site3-R: 5'-GGAAAATAATTGCAGAAAAG-3', Site4-F: 5'-TTGATGAAAATTGAAAATCA-3', Site-4-R: 5'-CACTTGGCTTCTTAAGATGT-3'.

\section{Animal study}

The 5 -week-old BALB/c nude mice were divided into four groups $(\mathrm{n}=5)$. Cells $\left(1 \times 10^{6}\right)$ with transfection were resuspended in $200 \mathrm{~mL}$ PBS and subcutaneously injected into the flanks of the mice. The lengths and widths of tumors were detected every week. Tumor volume was calculated as $=$ width $^{2} \times$ length $/ 2$. At 28 days, these tumors were excised and weighted. Experiments were performed under a project license (No. 2020KT042) granted by institutional/regional/national ethics/committee/ethics board of Affiliated Hospital 2 of Nantong University, in compliance with institutional guidelines for the care and use of animals.

\section{Statistical analysis}

Statistical analyses were conducted by SPSS 19.0. A student's $t$-test was implemented to analyze the differences between the two groups. Multiple comparisons between groups were employed by using ANOVA analysis. $\mathrm{P}$ value $<0.05$ was statistically significant.

\section{Results}

\section{Identification of clinical significance and expression of CAP2 in $G C$}

According to the criteria for screening DEGs, 642 genes that exhibited over-expression status in GC tissues were identified through the bioinformatics analysis of GSE84437 datasets. Among these genes, we included 172 candidate genes that were significantly connected with survival outcomes (log-rank $\mathrm{P}<0.001)$. Besides, univariate and multivariate Cox analysis was employed to select out 82 hub genes from these candidates, which can be performed to independently predict the survival time of GC patients. Then, we screened out the CAP2 gene from all hub genes and observed that the mRNA expression of CAP2 was increased in tumor tissues compared to the normal tissues (Figure 1A). Moreover, Kaplan-Meier plots showed that high CAP2 expression had a notably shorter overall survival time than GC patients in low CAP2 expression (Figure 1B). Also, elevated expression of CAP2 markedly associated with the tumor clinical stage (Figure 1C,D), suggesting that GC samples with high expression of CAP2 are prone to progress to a higher stage. Furthermore, we constructed the forest plot of univariate and multivariate analyses to assess the independent prognostic factors of CAP2. Cox's proportional hazards model demonstrated that CAP2 expression, T stage, and $\mathrm{N}$ stage were notably associated with the survival of GC patients (Figure 1E,F). CAP2 expression was further measured by western blot. Our results exhibited that the CAP2 protein level in tumor tissues was dramatically higher than that in matched normal tissues (Figure $2 A$ ). Taken together, our data identified that CAP2 could act as an oncogene and associated with GC patients' prognosis.

\section{Effects of CAP2 expression on the proliferation of GC cells}

To investigate the roles of CAP2 on GC cell proliferation, we performed the MTT and colony formation assays. Firstly, western blot analysis detected that CAP2 expression in MKN45 and BGC823 cells were higher than other GC cells and GES cells (Figure 2B). After transfected with three different CAP2 siRNA, we observed that siRNA1 had the highest knockdown efficiency compared with siRNA2 and siRNA3 by western blot (Figure 2C). Besides, the results indicated that the cell viability and the number of colonies 


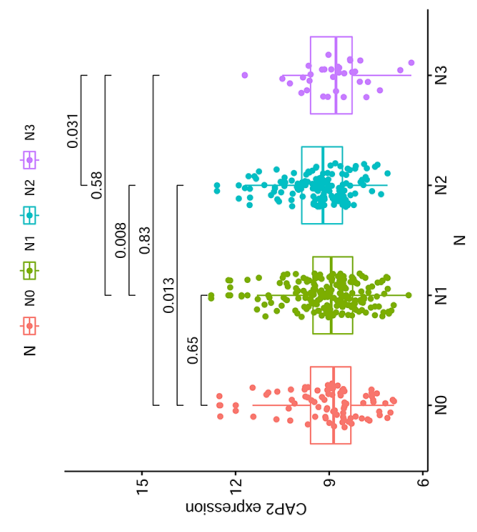

口

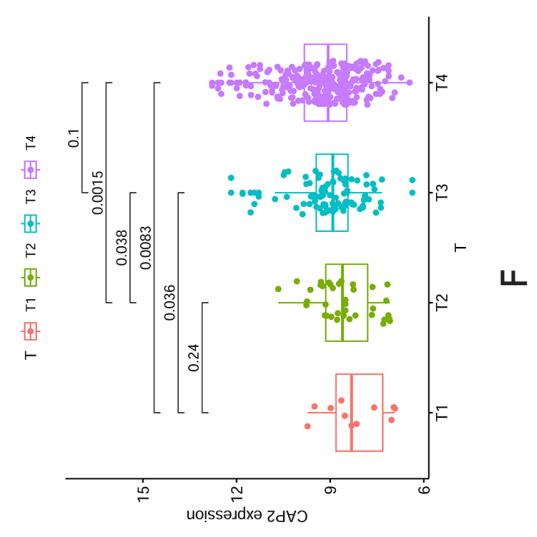

U
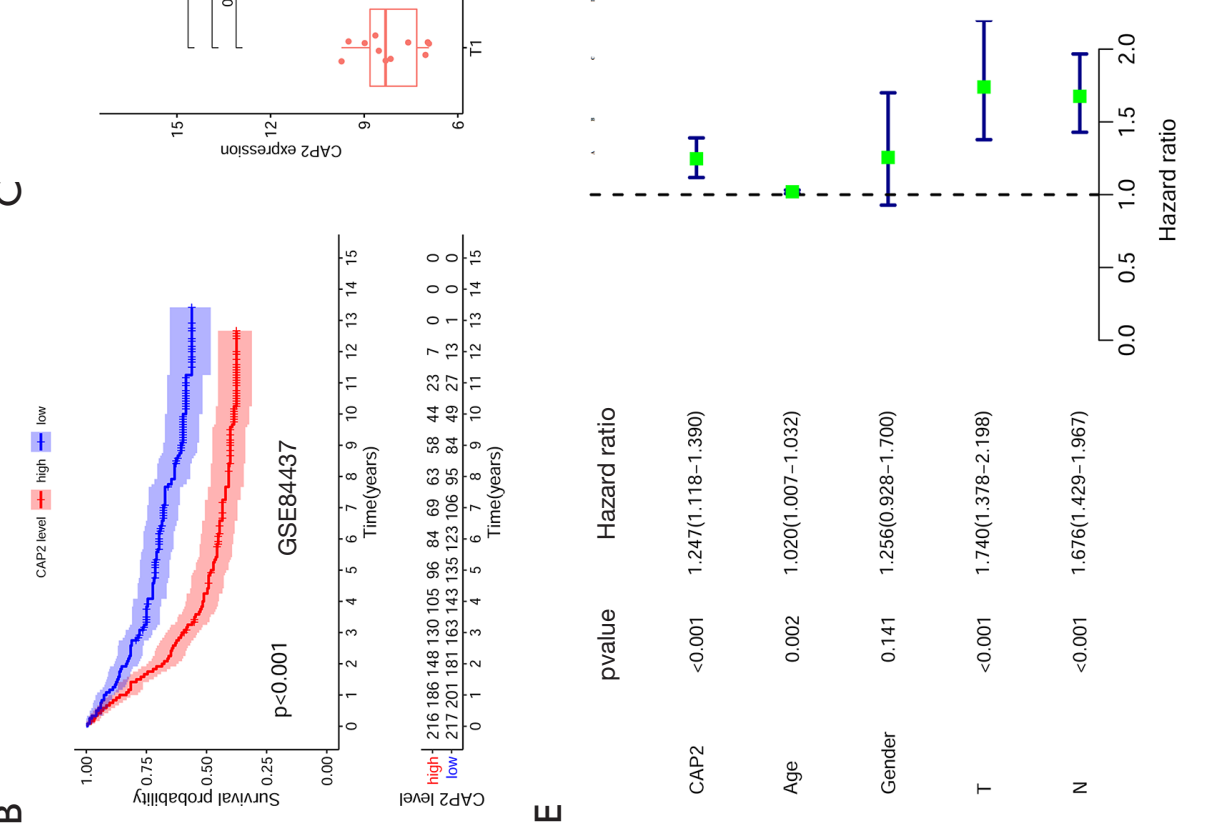

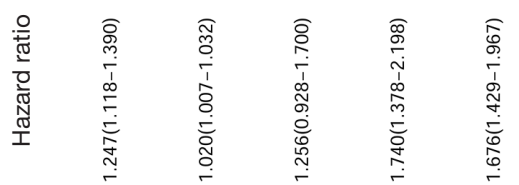

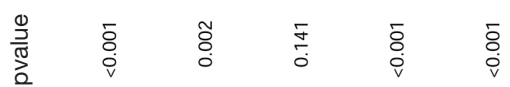

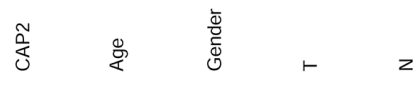

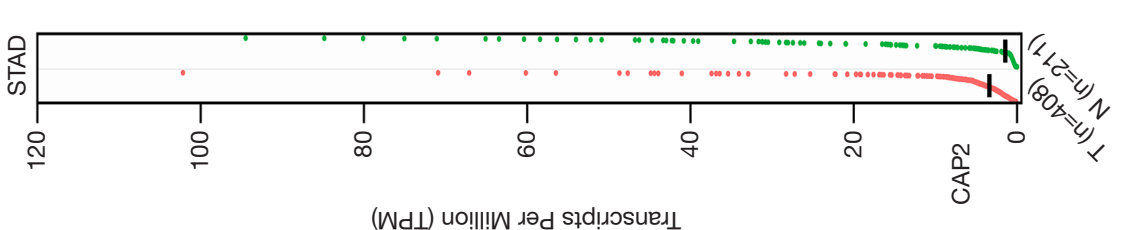

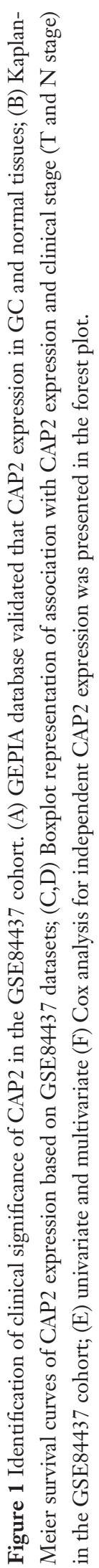


A
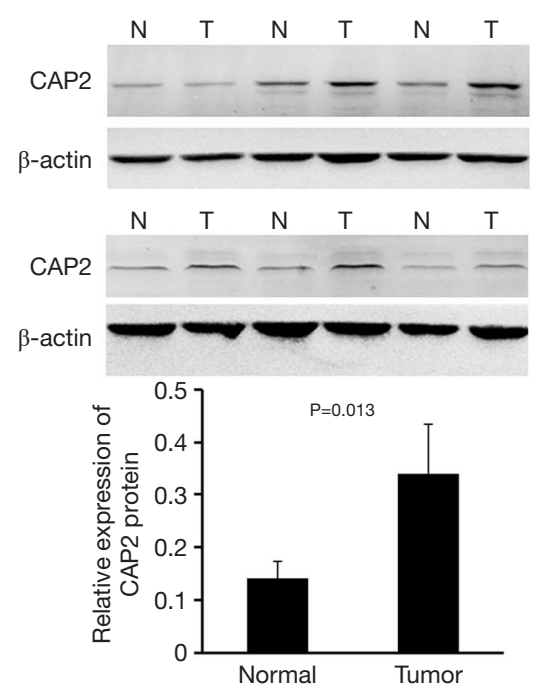

D

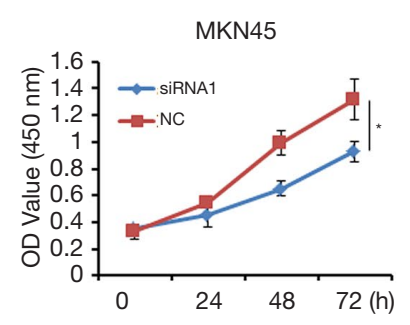

B
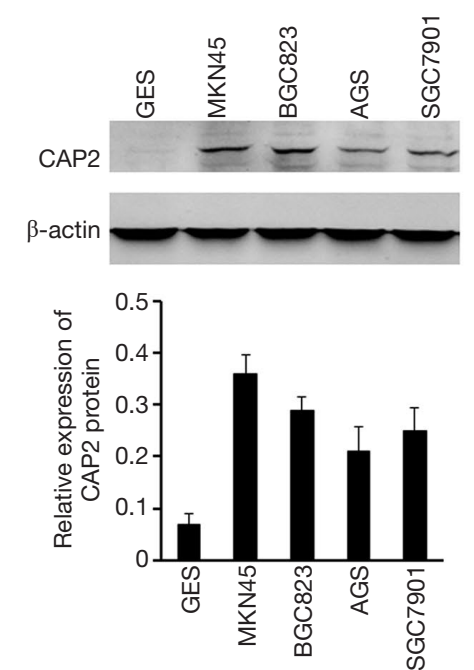

E
C

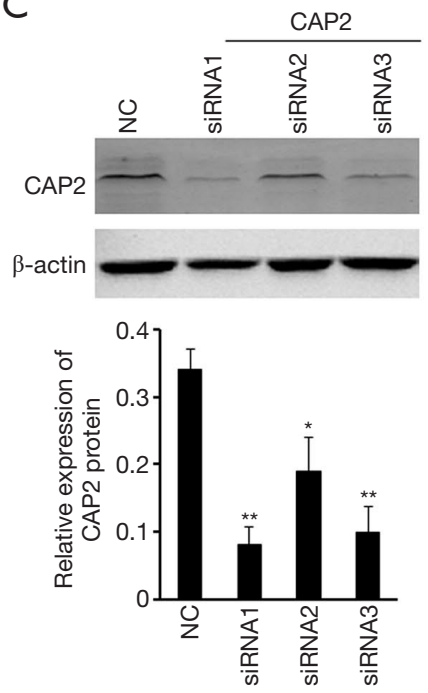

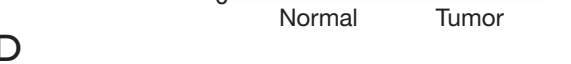

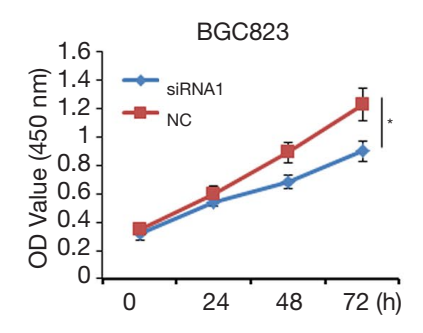

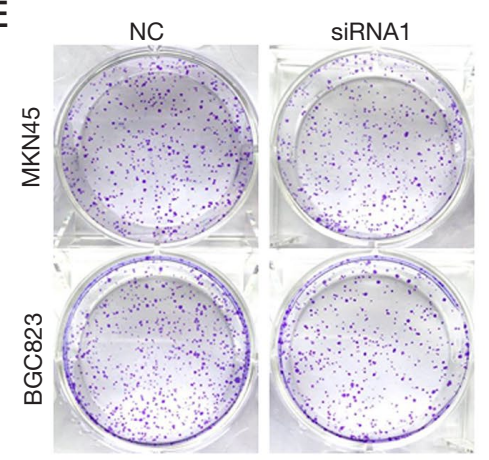

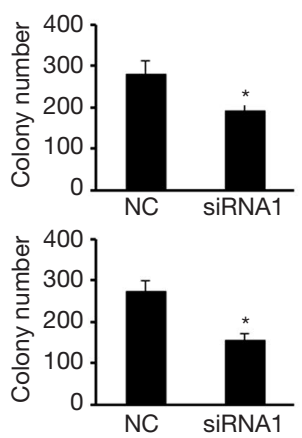

Figure 2 The expression of CAP2 is elevated in GC tissues and cells. (A) Representative western blot results of the protein levels of CAP2 in six pairs of GC tissues (T) and matched normal tissues (N); (B) the protein expression levels of CAP2 in GC cell lines and GES cell lines were detected by western blot; (C) Western blot analysis indicated the CAP2 expression level with three different CAP2 siRNA transfectants (siRNA1, siRNA2, and siRNA3) and the empty vector transfectant (NC); (D) effects of CAP2 expression on the cell viability of MKN45 and BGC823 cells transfected with CAP2 siRNA1 or sh-NC; (E) colony numbers of the treated GC cell lines were measured by colony formation assay. The colonies were stained with $1 \%$ crystal violet. *, $\mathrm{P}<0.05 ;{ }^{* *}, \mathrm{P}<0.01$.

on MKN45 and BGC823 cells in the siRNA1 treated group was dramatically reduced compared to the $\mathrm{NC}$ group (Figure 2D,E). Also, the EdU staining assay was utilized to assess the role of CAP2 on DNA replication of GC cells. The results showed that cell proliferation was observably suppressed in MKN45 and BGC823 cells transfected with the siRNA1 treated group (Figure $3 A, B$ ).

To further determine the effects of CAP2 on cell proliferation, we employed flow cytometry analysis to measure the cell cycle and apoptosis of GC cells after transfection with siRNA1. The results demonstrated that the percentage of cells in the G1 phase observably increased and the percentage of cells in the $\mathrm{S}$ phase dramatically decreased in MKN45 and BGC823 cells transfected with siRNA1 (Figure 3C). Besides, cell apoptosis analysis demonstrated that the apoptosis rate of cells was increased in MKN45 and BGC823 cells transfected with siRNA1 (Figure 3D). Therefore, these above results revealed that CAP2 could contribute to GC tumorigenesis.

\section{SOX9 is involved in CAP2-mediated proliferation of GC cells in vitro and in vivo}

To further explore the mechanisms that CAP2 is involved 


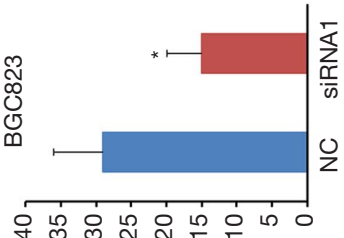

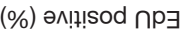
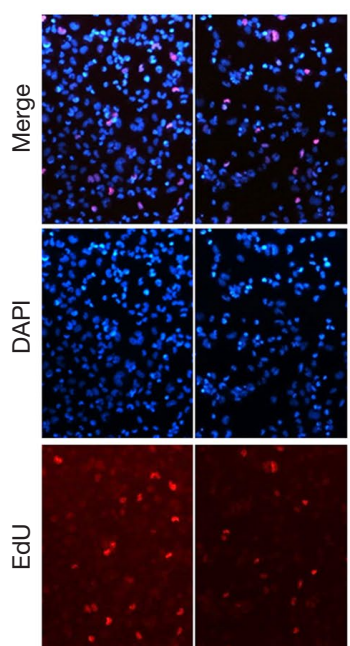

o

$\infty$
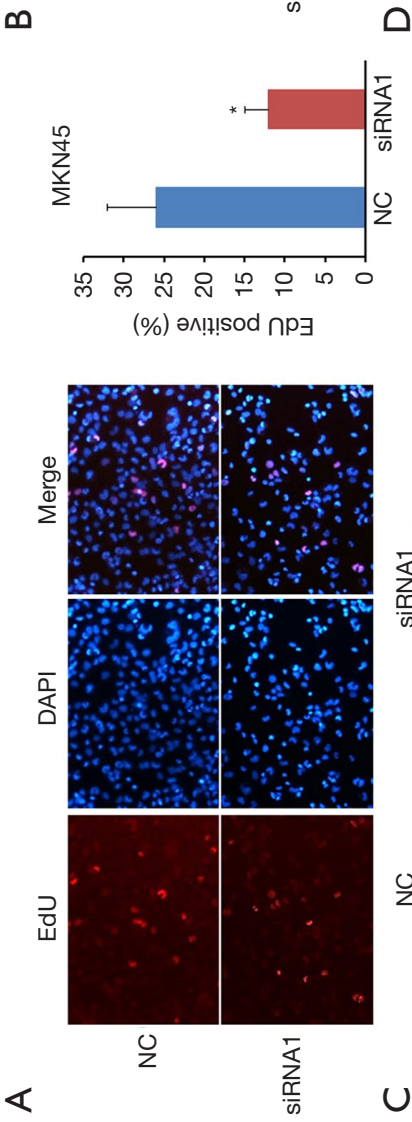

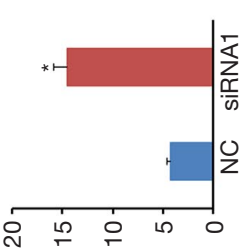

(\%) әㄹㅅ s!soldod $\forall$

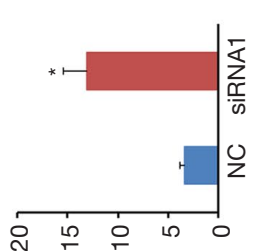

(\%) 핫 s!soldod $\forall$

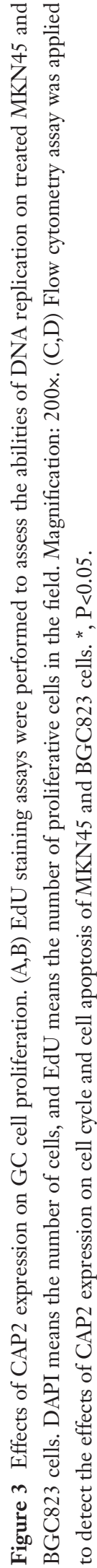


in GC tumorigenesis, we utilized the JASPAR database to estimate the possible binding sites of relevant transcription factors in the CAP2 promoter region. Our analysis showed that the possible binding sites for CAP2 within the -1200 to $-1192,-941$ to $-933,-714$ to -706 , and -567 to -559 regions (Figure $4 A$ ). ChIP assay was conducted to detect that the transcription factor SOX9 can be bind to the CAP2 promoter at Site-1, Site-2, Site-3, and Site-4 (Figure 4B). Then, we constructed serially truncated and mutated CAP2 promoter plasmids and conducted a dual-luciferase assay to present that the region from -1200 to -559 exhibited the strongest promoter activity, which seemed to be the core part explaining for increasing promoter activity (Figure 4C). To confirm whether the SOX9 binding sites take effects for CAP2 transcription, we performed substitution mutations of the sites (pGL3-M1, pGL3-M2, pGL3-M3, and pGL3-M4). We found that there was an observable decrease in CAP2 promoter activity when the -1200 to $-1192,-714$ to -706 , and -567 to -559 regions were respectively mutated. These data demonstrated that the SOX9 binding sites (Site1 , Site-3, and Site-4) are putative regulatory elements in the transcriptional activation of CAP2.

To further confirm that $\mathrm{SOX} 9$ can regulate the expression of CAP2, the results of western blot demonstrated that CAP2 expression in MKN45 and BGC823 cells transfected with SOX9 overexpression plasmid could notably upregulate the protein expression of CAP2. While silenced SOX9 expression by transfection of siSOX9 significantly downregulated CAP2 expression (Figure 4D,E). Next, western blot analysis revealed that overexpression of SOX9 enhanced the protein expression level of CAP2, and the effect was reversed by the siCAP2 transfection (Figure $4 F$ ). Moreover, we conducted a xenograft assay to determine the effects of SOX9 on CAP2-mediated tumor growth in vivo. Tumor xenograft results showed the effects that tumor volume and weight were significantly increased after transfection of SOX9 overexpression plasmid could be reversed by silenced CAP2 expression (Figure 4G). These above results suggested that SOX9 could play important roles in CAP2-mediated tumorigenesis in GC.

\section{Discussion}

Previously studies have indicated that CAP2 can regulate cellular actin dynamics to control cell migration and cell cycles (13). Overexpression of CAP2 can lead to tumor progression in various cancer types (14). Currently, it was reported that CAP2 was upregulated in breast cancer and was associated with patient survival, which could serve as a prognostic indicator for patients with breast cancer (11). In hepatocellular carcinoma, CAP2 was associated with cellular invasion and metastasis, and its high expression was significantly correlated with poor survival time (15). Moreover, CAP2 might play vital roles in the promotion of cell migration and proliferation of ovarian cancer, indicating that CAP2 could promote the progression of epithelial ovarian cancer (10). Additionally, CAP2 was significantly overexpressed in gliomas, and high CAP2 expression was notably related to the advanced stage (stage III and IV) (12). Furthermore, previous reports showed that CAP2 is overexpressed in malignant melanoma and its expression is linked to increase stepwise during melanoma progression (9). These above studies have demonstrated that CAP2 has been found to play a vital role in tumorigenesis. However, the mechanisms underlying CAP2 promotion of cancer metastasis remain largely unknown in GC.

In the present study, we identified one novel biomarker CAP2 from the GEO database (16), which might function as an oncogene in GC progression and associate with GC patients' prognosis. Subsequently, the expression level of CAP2 in GC tissues and cells was determined by western blot analysis. We further assessed the carcinogenic effects of CAP2 in GC, and our results revealed that the cell growth rate was markedly suppressed by CAP2 knockdown. Besides, by using colony formation assay and EdU assay, the results demonstrated that the number of colonies and the capability of DNA replication in GC cells were significantly decreased and inhibited by CAP2 knockdown. Furthermore, we found that knockdown of CAP2 could arrest cells in the G1 phase and play with the anti-apoptotic effect to regulate the survival of GC cells.

Various factors in the process of development are the key factors of tumorigenesis, including transcription factors (17). SOX transcription factors exert an important influence on tumor progression and metastasis (18). In addition, previous reports have established that as the high mobility group box transcription factor, SOX9 is overexpressed in GC (19), and associated with GC cell survival and promotes proliferation (20). Moreover, the molecular mechanisms for regulating the transcriptional activity of CAP2 by SOX9 to enhance GC cell proliferation have not been elucidated. In this study, we reported that SOX9 promoted the transcription of CAP2 through directly binding to its potential binding site. And SOX9 was demonstrated to be necessary for CAP2- 


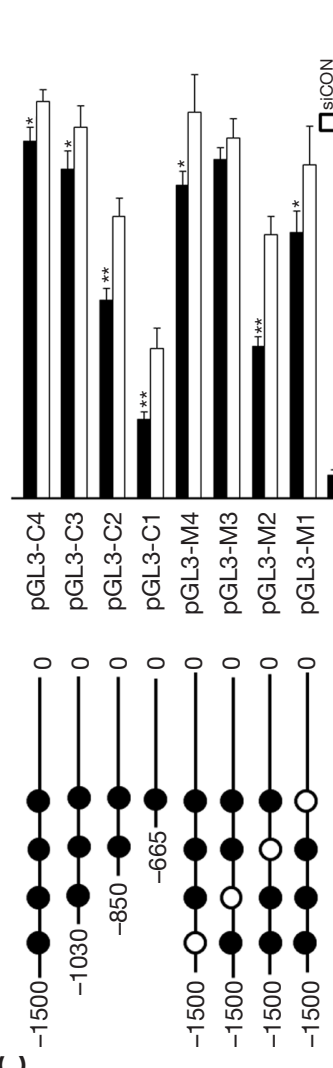

U

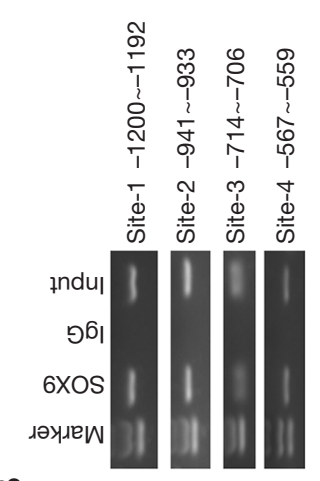

$\infty$

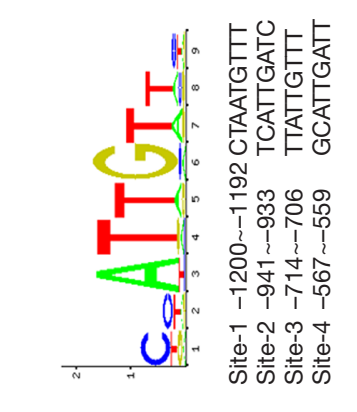

$\varangle$

ш
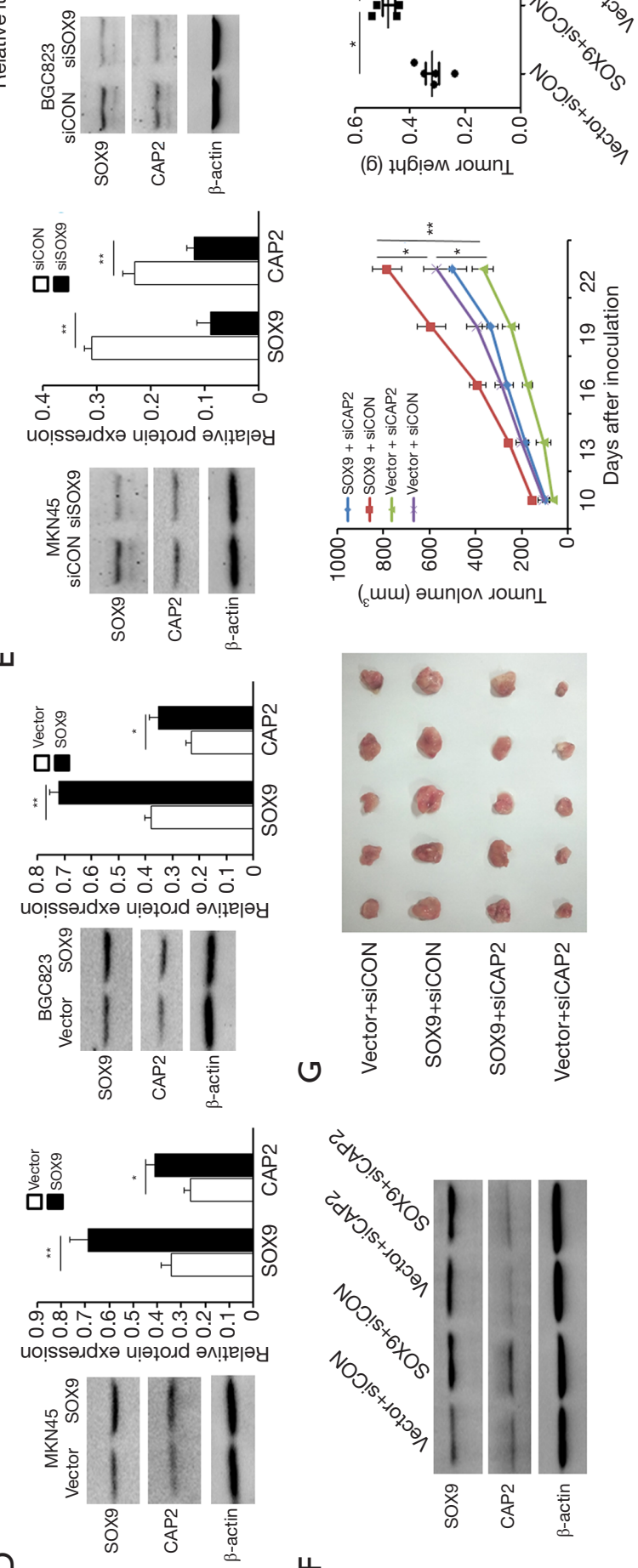

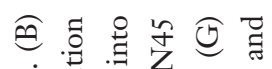

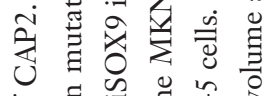
U.

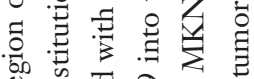

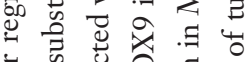

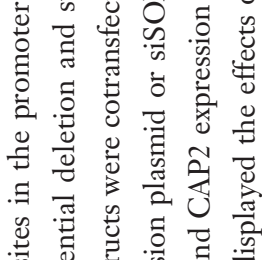
क्ष चี

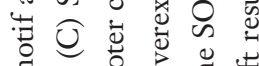
证 की 节焉艺 क

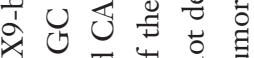
ᄋ.

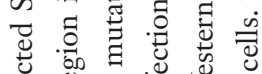

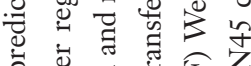

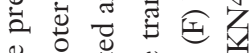

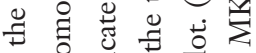

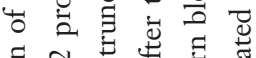
茟引艺芯

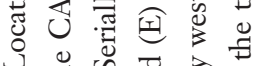

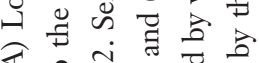

«

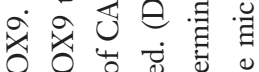

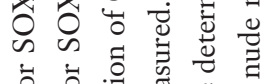

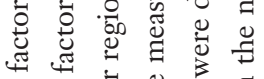

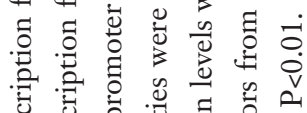

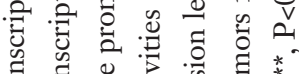

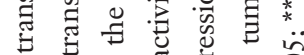

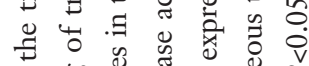

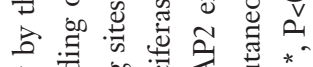

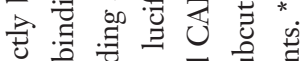

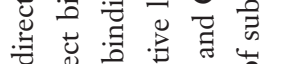
चु

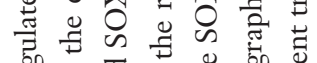

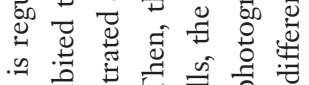

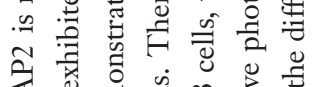

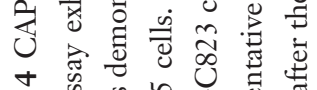

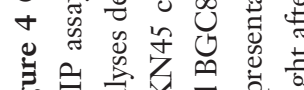

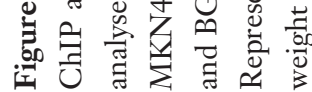


mediated GC proliferation in vitro and in vivo experiments. The above findings provide strong evidence to support the fact that CAP2 can have a significant impact on the genesis and development of GC.

\section{Conclusions}

In summary, our results identified that CAP2 is dramatically overexpressed in GC and involved in GC tumorigenesis. The silencing of CAP2 expression could inhibit the ability of cellular growth in vitro. Additionally, the effects of CAP2-mediated promotion of GC cell proliferation were influenced by positive transcriptional regulation of SOX9. So, our results not only provide novel insights into the precise molecular mechanism of CAP2 in GC tumorigenesis but also facilitate the development of future therapeutic strategies for GC.

\section{Acknowledgments}

Funding: This study was supported by grants of the Nantong Science and Technology Project (MS12018060 and QA2019003) and the Scientific Research Fund of Jiangsu Provincial Health Committee (H2019049).

\section{Footnote}

Reporting Checklist: The authors have completed the ARRIVE reporting checklist. Available at http://dx.doi. org/10.21037/jgo-20-234

Data Sharing Statement: Available at http://dx.doi. org/10.21037/jgo-20-234

Peer Review File: Available at http://dx.doi.org/10.21037/ jgo-20-234

Conflicts of Interest: All authors have completed the ICMJE uniform disclosure form (available at: http://dx.doi. org/10.21037/jgo-20-234). The authors have no conflicts of interest to declare.

Ethical Statement: The authors are accountable for all aspects of the work in ensuring that questions related to the accuracy or integrity of any part of the work are appropriately investigated and resolved. This study was conducted in accordance with the Declaration of Helsinki (as revised in 2013), and was approved by Ethics the
Committee of Affiliated Hospital 2 of Nantong University (ID: 2020KT042). Informed consent was provided by all participants. Experiments were performed under a project license (No. 2020KT042) granted by institutional/regional/ national ethics/committee/ethics board of Affiliated Hospital 2 of Nantong University, in compliance with institutional guidelines for the care and use of animals.

Open Access Statement: This is an Open Access article distributed in accordance with the Creative Commons Attribution-NonCommercial-NoDerivs 4.0 International License (CC BY-NC-ND 4.0), which permits the noncommercial replication and distribution of the article with the strict proviso that no changes or edits are made and the original work is properly cited (including links to both the formal publication through the relevant DOI and the license). See: https://creativecommons.org/licenses/by-nc-nd/4.0/.

\section{References}

1. Van Cutsem E, Sagaert X, Topal B, et al. Gastric cancer. Lancet 2016;388:2654-64.

2. Ma Z, Chen M, Yang X, et al. The role of cancerassociated fibroblasts in tumorigenesis of gastric cancer. Curr Pharm Des 2018;24:3297-302.

3. Bray F, Ferlay J, Soerjomataram I, et al. Global cancer statistics 2018: GLOBOCAN estimates of incidence and mortality worldwide for 36 cancers in 185 countries. CA Cancer J Clin 2018;68:394-424.

4. Nagini S. Carcinoma of the stomach: A review of epidemiology, pathogenesis, molecular genetics and chemoprevention. World Journal of Gastrointestinal Oncology 2012;4:156-69.

5. Makkonen M, Bertling E, Chebotareva NA, et al. Mammalian and Malaria Parasite Cyclase-associated Proteins Catalyze Nucleotide Exchange on G-actin through a Conserved Mechanism. J Biol Chem 2013;288:984-94.

6. Olson MF, Sahai E. The actin cytoskeleton in cancer cell motility. Clin Exp Metastasis 2009;26:273-87.

7. Hubberstey A, Yu G, Loewith R, et al. Mammalian CAP interacts with CAP, CAP2, and actin. J Cell Biochem 1996;61:459-66.

8. Chen M, Zheng T, Han S, et al. A preliminary study of plasma cyclase-associated protein 2 as a novel biomarker for early stage and alpha-fetoprotein negative hepatocellular carcinoma patients. Clinics and Research in Hepatology and Gastroenterology 2015;39:215-21. 
9. Masugi Y, Tanese K, Emoto K, et al. Overexpression of adenylate cyclase-associated protein 2 is a novel prognostic marker in malignant melanoma. Pathol Int 2015;65:627-34.

10. Adachi M, Masugi Y, Yamazaki K, et al. Upregulation of cyclase-associated actin cytoskeleton regulatory protein 2 in epithelial ovarian cancer correlates with aggressive histologic types and worse outcomes. Jpn J Clin Oncol 2020;50:643-52.

11. Xu L, Peng S, Huang Q, et al. Expression status of cyclase associated protein 2 as a prognostic marker for human breast cancer. Oncol Rep 2016;36:1981-8.

12. Saker Z, Bahmad H, Fares Y, et al. Prognostic impact of adenylyl cyclase-associated protein 2 (CAP2) in glioma: A clinicopathological study. Heliyon 2020;6:e03236.

13. Ono $\mathrm{S}$. The role of cyclase-associated protein in regulating actin filament dynamics - more than a monomersequestration factor. J Cell Sci 2013;126:3249-58.

14. Peche V, Shekar S, Leichter M, et al. CAP2, cyclaseassociated protein 2, is a dual compartment protein. Cell

Cite this article as: Wan Y, Qiu S, Yin L, Gao X, Jiang Y, Feng S, Tang C. CAP2 contributes to tumorigenesis in gastric cancer by targeting transcription factor SOX9. J Gastrointest Oncol 2021;12(2):268-277. doi: 10.21037/jgo-20-234
Mol Life Sci 2007;64:2702-15.

15. Effendi K, Yamazaki K, Mori T, et al. Involvement of hepatocellular carcinoma biomarker, cyclase-associated protein 2 in zebrafish body development and cancer progression. Exp Cell Res 2013;319:35-44.

16. Barrett T, Wilhite SE, Ledoux P, et al. NCBI GEO: archive for functional genomics data sets--update. Nucleic Acids Res 2013;41:D991-995.

17. Grimm D, Bauer J, Wise P, et al. The role of SOX family members in solid tumours and metastasis. Semin Cancer Biol 2020;67:122-53.

18. She ZY, Yang WX. SOX family transcription factors involved in diverse cellular events during development. Eur J Cell Biol 2015;94:547-63.

19. Matheu A, Collado M, Wise C, et al. Oncogenicity of the Developmental Transcription Factor Sox9. Cancer Res 2012;72:1301-15.

20. Santos JC, Carrascogarcia E, Garciapuga M, et al. SOX9 Elevation Acts with Canonical WNT Signaling to Drive Gastric Cancer Progression. Cancer Res 2016;76:6735-46. 